\title{
A sufficient condition for pre-Hamiltonian cycles in bipartite digraphs
}

\author{
Samvel Kh. Darbinyan and Iskandar A. Karapetyan \\ Institute for Informatics and Automation Problems, Armenian National Academy of Sciences \\ E-mails: samdarbin@ipia.sci.am, isko@ipia.sci.am
}

\begin{abstract}
Let $D$ be a strongly connected balanced bipartite directed graph of order $2 a \geq 10$ other than a directed cycle. Let $x, y$ be distinct vertices in $D$. $\{x, y\}$ dominates a vertex $z$ if $x \rightarrow z$ and $y \rightarrow z$; in this case, we call the pair $\{x, y\}$ dominating. In this paper we prove:

If $\max \{d(x), d(y)\} \geq 2 a-2$ for every dominating pair of vertices $\{x, y\}$, then $D$ contains cycles of all lengths $2,4, \ldots, 2 a-2$ or $D$ is isomorphic to a certain digraph of order ten which we specify.
\end{abstract}

Keywords: Digraphs, pre-Hamiltonian cycles, bipartite balanced digraphs, even pancyclic.

\section{Introduction}

It is sequel to the paper [13] by the first author. We consider digraphs (directed graphs) in the sense of [4], and use standard graph theoretical terminology and notation (see Section 2 for details). A cycle passing through all the vertices of a digraph is called Hamiltonian. A digraph containing a Hamiltonian cycle is called a Hamiltonian digraph. A digraph $D$ of order $n$ is called pancyclic if it contains cycles of every lengths $3,4, \ldots, n$. Various sufficient conditions for a digraph to be Hamiltonian have been given in terms of the vertex degree of the digraph. Here we recall some of them which are due to Ghouila-Houri [16], Nash-Williams [23], Woodall [27], Meyniel [22], Thomassen [25] and Darbinyan [11]. The Meyniel theorem is a generalization Nash-Williams', Ghouila-Houri's and Woodall's theorems.

Bondy suggested (see [9] by Chvátal) the following metaconjecture:

Metaconjecture. Almost any non-trivial condition of a graph (digraph) which implies that the graph (digraph) is Hamiltonian also implies that the graph (digraph) is pancyclic. (There may be a "simple" family of exceptional graphs (digraphs)).

In fact various sufficient conditions for a digraph to be Hamiltonian are also sufficient for the digraph to be pancyclic. Namely, in $[20,24,10,12]$, it was shown that if a digraph $D$ satisfies one of the above mentioned conditions for hamiltonicity digraphs, then the digraph $D$ also is pancyclic (unless some extremal cases which are characterized). For additional information on Hamiltonian and pancyclic digraphs, see, e.g, the book by Bang-Jensen and Gutin [4] and the surveys [7] by Bermond and Thomassen, [21] by Kühn and Ostus and [17] by Gutin.

Each of aforementioned theorems imposes a degree condition on all vertices (or, on all pairs of nonadjacent vertices). In [5] and [3], it was described a type of sufficient conditions for a digraph to be Hamiltonian, in which a degree condition requires only for some pairs of nonadjacent vertices. Let us recall only the following theorem of them.

Theorem 1.1 (Bang-Jensen, Gutin, H.Li [5]). Let D be a strongly connected digraph of order $n \geq 2$. Suppose that $\min \{d(x), d(y)\} \geq n-1$ and $d(x)+d(y) \geq 2 n-1$ for any pair of non-adjacent vertices $x, y$ 
with a common in-neighbour. Then $D$ is Hamiltonian.

A digraph $D$ is called a bipartite digraph if there exists a partition $X, Y$ of its vertex set into two partite sets such that every arc of $D$ has its end-vertices in different partite sets. It is called balanced if $|X|=|Y|$.

There are analogies results to the Nash-Williams, Ghouila-Houri, Woodall, Meyniel and Thomassen theorems for balanced bipartite digraphs (see e.g., [2] and the papers cited there).

An analogue of Theorem 1.1 for bipartite digraphs was given by R. Wang [26] and recently a different result was given by Adamus [1].

Theorem 1.2 (R. Wang [26]). Let $D$ be a strongly connected balanced bipartite digraph of order $2 a$, where $a \geq 1$. Suppose that, for every dominating pair of vertices $\{x, y\}$, either $d(x) \geq 2 a-1$ and $d(y) \geq a+1$ or $d(y) \geq 2 a-1$ and $d(x) \geq a+1$. Then $D$ is Hamiltonian.

Theorem 1.3 (Adamus [1]). Let $D$ be a strongly connected balanced bipartite digraph of order $2 a$, where $a \geq 3$. If $d(x)+d(y) \geq 3$ a for every pair of vertices $\{x, y\}$ with a common in-neighbour or a common out-neighbour, then $D$ is Hamiltonian.

Let $D$ be a balanced bipartite digraph of order $2 a$, where $a \geq 2$. For integer $k \geq 0$, we say that $D$ satisfies condition $B_{k}$ when $\max \{d(x), d(y)\} \geq 2 a-2+k$ for every pair of dominating vertices $x$ and $y$.

Before stating the next theorems we need to define three digraphs.

Example 1. Let $D(10)$ be a bipartite digraph with partite sets $X=\left\{x_{0}, x_{1}, x_{2}, x_{3}, x_{4}\right\}$ and $Y=$ $\left\{y_{0}, y_{1}, y_{2}, y_{3}, y_{4}\right\}$ satisfying the following conditions: The induced subdigraph $\left\langle\left\{x_{1}, x_{2}, x_{3}, y_{0}, y_{1}\right\}\right\rangle$ is a complete bipartite digraph with partite sets $\left\{x_{1}, x_{2}, x_{3}\right\}$ and $\left\{y_{0}, y_{1}\right\} ;\left\{x_{1}, x_{2}, x_{3}\right\} \rightarrow\left\{y_{2}, y_{3}, y_{4}\right\} ; x_{4} \leftrightarrow y_{1}$; $x_{0} \leftrightarrow y_{0}$ and $x_{i} \leftrightarrow y_{i+1}$ for all $i \in[1,3]$. $D(10)$ contains no other arcs.

It is easy to check that the digraph $D(10)$ is strongly connected and satisfies condition $B_{0}$, but the underlying undirected graph of $D(10)$ is not 2-connected and $D(10)$ has no cycle of length 8 . (It follows from the facts that $d\left(x_{0}\right)=d\left(x_{4}\right)=2$ and $x_{0}\left(x_{4}\right)$ is on 2-cycle). It is not difficult to check that any digraph obtained from $D(10)$ by adding a new arc whose one end-vertex is $x_{0}$ or $x_{4}$ contains no cycle of length eight. Moreover, if to $A(D)$ we add some new $\operatorname{arcs}$ of the type $y_{i} x_{j}$, where $i \in[2,4]$ and $j \in[1,3]$, then always we obtain a digraph which does not satisfy condition $B_{0}$.

Example 2. Let $K_{2,3}^{*}$ be a complete bipartite digraph with partite sets $\left\{x_{1}, x_{2}\right\}$ and $\left\{y_{1}, y_{2}, y_{3}\right\}$. Let $H(8)$ be the bipartite digraph obtained from the digraph $K_{2,3}^{*}$ by adding three new vertices $x_{0}, y_{0}, x_{3}$ and the following new arcs $x_{0} y_{0}, y_{0} x_{0}, x_{0} y_{1}, y_{1} x_{0}, x_{3} y_{3}$ and $y_{3} x_{3}$.

It is not difficult to check that the digraph $H(8)$ is strongly connected and satisfies condition $B_{0}$, but the underlying undirected graph of $H(8)$ is not 2-connected and $H(8)$ has no cycle of length 6 . Hence, the bound on order of $D$ in Theorem 3.4 is sharp.

Example 3. Let $D(8)$ be a bipartite digraph with partite sets $X=\left\{x_{0}, x_{1}, x_{2}, x_{3}\right\}$ and $Y=$ $\left\{y_{0}, y_{1}, y_{2}, y_{3}\right\}$, and the arc set $A(D(8))$ contains exactly the following arcs $y_{0} x_{1}, y_{1} x_{0}, x_{2} y_{3}, x_{3} y_{2}$ and all the arcs of the following 2-cycles: $x_{i} \leftrightarrow y_{i}, i \in[0,3], y_{0} \leftrightarrow x_{2}, y_{0} \leftrightarrow x_{3}, y_{1} \leftrightarrow x_{2}$ and $y_{1} \leftrightarrow x_{3}$.

It is easy to see that

$$
d\left(x_{2}\right)=d\left(x_{3}\right)=d\left(y_{0}\right)=d\left(y_{1}\right)=7 \quad \text { and } \quad d\left(x_{0}\right)=d\left(x_{1}\right)=d\left(y_{2}\right)=d\left(y_{3}\right)=3,
$$

and the dominating pairs in $D(8)$ are: $\left\{y_{0}, y_{1}\right\},\left\{y_{0}, y_{2}\right\},\left\{y_{0}, y_{3}\right\},\left\{y_{1}, y_{2}\right\},\left\{y_{1}, y_{3}\right\},\left\{x_{0}, x_{2}\right\},\left\{x_{0}, x_{3}\right\}$, 
$\left\{x_{1}, x_{2}\right\},\left\{x_{1}, x_{3}\right\}$ and $\left\{x_{2}, x_{3}\right\}$. Note that every dominating pair satisfies condition $B_{1}$. Since $x_{0} y_{0} x_{3} y_{2} x_{2}$ $y_{1} x_{0}$ is a cycle of length 6 in $D(8)$, it is not difficult to check that $D(8)$ is strong.

Observe that $D(8)$ is not Hamiltonian. Indeed, if $D(8)$ contains a Hamiltonian cycle, say $C$, then $C$ would contain the $\operatorname{arcs} x_{1} y_{1}$ and $x_{0} y_{0}$. Therefore, $C$ must contain the path $x_{1} y_{1} x_{0} y_{0}$ or the path $x_{0} y_{0} x_{1} y_{1}$, which is impossible since $N^{-}\left(x_{0}\right)=N^{-}\left(x_{1}\right)=\left\{y_{0}, y_{1}\right\}$.

For $a \geq 5$ Theorem 1.2 is an immediate consequence of the following theorem by the first author [14].

Theorem 1.4 (Darbinyan [14]). Let $D$ be a strongly connected balanced bipartite digraph of order $2 a$, where $a \geq 4$. Suppose that, for every dominating pair of vertices $\{x, y\}$, either $d(x) \geq 2 a-1$ or $d(y) \geq 2 a-1$. Then either $D$ is Hamiltonian or isomorphic to the digraph $D(8)$.

A balanced bipartite digraph of order $2 m$ is even pancyclic if it contains a cycle of length $2 k$ for any $2 \leq k \leq m$. A cycle of a balanced bipartite digraph $D$ is called pre-Hamiltonian if it contains all the vertices of $D$ except two.

Characterizations of even pancyclic bipartite tournaments was given in [6] and [28]. A characterization of pancyclic ordinary $k$-partite $(k \geq 3)$ tournaments (respectively, pancyclic ordinary complete multipartite digraphs) was established in [18] (respectively, in [19]).

Motivated by the Bondy's metaconjecture, it is natural to set the following problem:

Problem. Characterize those digraphs which satisfy the conditions of Theorem 1.2 (or, 1.3 or 1.4) but are not even pancyclic.

In [15], the first author have proved the following Theorems 1.5 and 1.6.

Theorem 1.5 ([15]. Let $D$ be a strongly connected balanced bipartite digraph of order $2 a \geq 8$ other than a directed cycle. If $\max \{d(x), d(y)\} \geq 2 a-1$ for every dominating pair of vertices $\{x, y\}$, then $D$ contains a cycles of all even lengths less than equal $2 a$ or $D$ is isomorphic to the digraph $D(8)$.

Theorem 1.6. ([15]. Let $D$ be a strongly connected balanced bipartite digraph of order $2 a \geq 8$ which contains a cycle of length $2 a-2$. If $\max \{d(x), d(y)\} \geq 2 a-2$ for every dominating pair of vertices $\{x, y\}$, then for any $k, 1 \leq k \leq a-1, D$ contains a cycle of length $2 k$.

In view of Theorem 1.6 it seems quite natural to ask whether a balanced bipartite digraph of order $2 a$, which satisfies condition $B_{0}$ contains a pre-Hamiltonian cycle (i.e., a cycle of length $2 a-2$ ). In this paper we prove the following theorems.

Theorem 1.7. Let $D$ be a strongly connected balanced bipartite digraph of order $2 a \geq 10$ other than the directed cycle of length $2 a$. Suppose that $D$ satisfies condition $B_{0}$, i.e., $\max \{d(x), d(y)\} \geq 2 a-2$ for every dominating pair of vertices $\{x, y\}$. Then $D$ contains a cycle of lengths $2 a-2$ unless $D$ is isomorphic to the digraph $D(10)$.

From Theorem 1.6 and 1.7 it follows the following theorem.

Theorem 1.8. Let $D$ be a balanced bipartite digraph of order $2 a \geq 10$ other than the directed cycle of length $2 a$. Suppose that $D$ satisfies condition $B_{0}$, i.e., $\max \{d(x), d(y)\} \geq 2 a-2$ for every dominating pair of vertices $\{x, y\}$. Then $D$ contains cycles of all lengths $2,4, \ldots, 2 a-2$ unless $D$ is isomorphic to the digraph $D(10)$. 


\section{Terminology and Notation}

In this paper we consider finite digraphs without loops and multiple arcs. The vertex set and the arc set of a digraph $D$ are denoted by $V(D)$ and by $A(D)$, respectively. The order of $D$ is the number of its vertices. For any $x, y \in V(D)$, we also write $x \rightarrow y$, if $x y \in A(D)$. If $x y \in A(D)$, then we say that $x$ dominates $y$ or $y$ is an out-neighbour of $x$, and $x$ is an in-neighbour of $y$. The notation $x \leftrightarrow y$ denotes that $x \rightarrow y$ and $y \rightarrow x$ ( $x \leftrightarrow y$ is called a 2-cycle). We denote by $a(x, y)$ the number of arcs with end-vertices $x$ and $y$. For disjoint subsets $A$ and $B$ of $V(D)$ we define $A(A \rightarrow B)$ as the set $\{x y \in A(D) \mid x \in A, y \in B\}$ and $A(A, B)=A(A \rightarrow B) \cup A(B \rightarrow A)$. If $x \in V(D)$ and $A=\{x\}$ we write $x$ instead of $\{x\}$. If $A$ and $B$ are two disjoint subsets of $V(D)$ such that every vertex of $A$ dominates every vertex of $B$, then we say that $A$ dominates $B$, denoted by $A \rightarrow B$. $A \mapsto B$ means that $A \rightarrow B$ and there is no arc from $B$ to $A$.

We let $N^{+}(x), N^{-}(x)$ denote the set of out-neighbours, respectively the set of in-neighbours of a vertex $x$ in a digraph $D$. If $A \subseteq V(D)$, then $N^{+}(x, A)=A \cap N^{+}(x)$ and $N^{-}(x, A)=A \cap N^{-}(x)$. The out-degree of $x$ is $d^{+}(x)=\left|N^{+}(x)\right|$ and $d^{-}(x)=\left|N^{-}(x)\right|$ is the in-degree of $x$. Similarly, $d^{+}(x, A)=\left|N^{+}(x, A)\right|$ and $d^{-}(x, A)=\left|N^{-}(x, A)\right|$. The degree of a vertex $x$ in $D$ is defined as $d(x)=d^{+}(x)+d^{-}(x)$ (similarly, $\left.d(x, A)=d^{+}(x, A)+d^{-}(x, A)\right)$.

For integers $a$ and $b, a \leq b$, let $[a, b]$ denote the set of all integers which are not less than $a$ and are not greater than $b$.

The subdigraph of $D$ induced by a subset $A$ of $V(D)$ is denoted by $D\langle A\rangle$ or $\langle A\rangle$ for brevity. The path (respectively, the cycle) consisting of the distinct vertices $x_{1}, x_{2}, \ldots, x_{m}(m \geq 2)$ and the $\operatorname{arcs} x_{i} x_{i+1}$, $i \in[1, m-1]$ (respectively, $x_{i} x_{i+1}, i \in[1, m-1]$, and $x_{m} x_{1}$ ), is denoted by $x_{1} x_{2} \cdots x_{m}$ (respectively, $\left.x_{1} x_{2} \cdots x_{m} x_{1}\right)$. We say that $x_{1} x_{2} \cdots x_{m}$ is a path from $x_{1}$ to $x_{m}$ or is an $\left(x_{1}, x_{m}\right)$-path. If $P$ is a path containing a subpath from $x$ to $y$ we let $P[x, y]$ denote that subpath. Similarly, if $C$ is a cycle containing vertices $x$ and $y, C[x, y]$ denotes the subpath of $C$ from $x$ to $y$. A digraph $D$ is strongly connected (or, just, strong) if there exists an $(x, y)$-path in $D$ for every ordered pair of distinct vertices $x, y$ of $D$. Given a vertex $x$ of a path $P$ or a cycle $C$, we denote by $x^{+}$(respectively, by $x^{-}$) the successor (respectively, the predecessor) of $x$ (on $P$ or $C$ ), and in case of ambiguity, we precise $P$ or $C$ as a subscript (that is $x_{P}^{+}$ ...). Two distinct vertices $x$ and $y$ are adjacent if $x y \in A(D)$ or $y x \in A(D)$ (or both).

Let $C$ be a non-Hamiltonian cycle in a digraph $D$. An $(x, y)$-path $P$ is a $C$-bypass if $|V(P)| \geq 3$, $x \neq y$ and $V(P) \cap V(C)=\{x, y\}$. The length of the path $C[x, y]$ is the gap of $P$ with respect to $C$.

The underlying undirected graph of a digraph $D$ is the unique graph that contains an edge $x y$ if $x \rightarrow y$ or $y \rightarrow x$ (or both).

\section{$3 \quad$ Preliminaries}

Lemma 3.1 (Bypass Lemma 3.17, Bondy [8]). Let $D$ be a strongly connected digraph, and let $H$ be a non-trivial proper subdigraph of $D$. If the underlying undirected graph of $D$ is 2-connected, then $D$ contains a $H$-bypass.

Remark. One can prove Bypass Lemma using the proof of Theorem 5.4.2 [4].

Lemma 3.2 ([14]). Let $D$ be a strongly connected balanced bipartite digraph of order $2 a \geq 8$ other than the directed cycle of length $2 a$. If $D$ satisfies condition $B_{0}$, then $D$ contains a non-Hamiltonian cycle of length at least 4 .

Lemma $3.3([15])$. Let $D$ be a strongly connected balanced bipartite digraph of order $2 a \geq 8$ with partite sets $X$ and $Y$. Assume that $D$ satisfies condition $B_{0}$. Let $C=x_{1} y_{1} x_{2} y_{2} \ldots x_{k} y_{k} x_{1}$ be a longest 
non-Hamiltonian cycle in $D$, where $k \geq 2, x_{i} \in X$ and $y_{i} \in Y$, and $P$ be a C-bypass. If the gap of $P$ with respect to $C$ is equal to one, then $k=a-1$, i.e., the longest non-Hamiltonian cycle in $D$ has length $2 a-2$.

Theorem 3.4 ([13]). Let $D$ be a strongly connected balanced bipartite digraph of order $2 a \geq 10$. Assume that $D$ satisfies condition $B_{0}$. Then either the underlying undirected graph of $D$ is 2-connected or $D$ contains a cycle of length $2 a-2$ unless $D$ is isomorphic to the digraph $D(10)$.

\section{$4 \quad$ Proof of the main result}

Proof of Theorem 1.7. Suppose, on the contrary, that a digraph $D$ is not a directed cycle and satisfies the conditions of the theorem, but contains no cycle of length $2 a-2$. Let $C=x_{1} y_{1} x_{2} y_{2} \ldots x_{m} y_{m} x_{1}$ be a longest non-Hamiltonian cycle in $D$, where $x_{i} \in X$ and $y_{i} \in Y$ (all subscripts of the vertices $x_{i}$ and $y_{i}$ are taken modulo $m$, i.e., $x_{m+i}=x_{i}$ and $y_{m+i}=y_{i}$ ).

By Lemma 3.2, $D$ contains a non-Hamiltonian cycle of length at least 4 , i.e., $2 \leq m \leq a-2$. By Theorem 3.4, the underlying undirected graph of $D$ is 2-connected. Therefore, by Bypass Lemma, $D$ contains a $C$-bypass. We choose a cycle $C$ and a $C$-bypass $P:=x u_{1} \ldots u_{s} y$ such that

(i) $C$ is a longest non-Hamiltonian cycle in $D$;

(ii) the gap of $C$-bypass $P$ with respect to $C$ is minimum subject to (i);

(iii) the length of $P$ is minimum subject to (i) and (ii).

Without loss of generality, we assume that $x:=x_{1}$. Let $R:=V(D) \backslash V(C), P_{1}:=P\left[u_{1}, u_{s}\right]$ and $C^{\prime}:=V\left(C\left[y_{1}, y_{C}^{-}\right]\right)$. Note that $|R| \geq 4$. From Lemma 3.3 it follows that $|V(C[x, y])| \geq 3$. Then $\left|C^{\prime}\right| \geq s$ since $C$ is a longest non-Hamiltonian cycle in $D$.

We first prove that $\left|V\left(P_{1}\right)\right|=1$, i.e., $s=1$.

Proof of $s=1$. Suppose, on the contrary, that $s \geq 2$. By the minimality of the gap $\left|C^{\prime}\right|+1$, we have

$$
d\left(y_{C}^{-}, P_{1}\right)=d\left(u_{s}, C^{\prime}\right)=0 .
$$

It is not difficult to see that $s \leq 3$. Indeed, if $s \geq 4$, then from (1) it follows that $d\left(y_{C}^{-}\right) \leq 2 a-4$ and $d\left(u_{s}\right) \leq 2 a-4$, since each of $P_{1}$ and $C^{\prime}$ contains at least two vertices from each partite set. So, we have a contradiction, since $\left\{u_{s}, y_{C}^{-}\right\} \rightarrow y$. Therefore, $s=3$ or $s=2$.

First consider the case $s=3$, i.e., $P_{1}=u_{1} u_{2} u_{3}$. From our assumption that $x=x_{1}$ it follows that $u_{1}, u_{3} \in Y$ and $y \in X$. Therefore, $u_{2} \in X$ and $y_{C}^{-} \in Y$. Note that $x_{2} \in C^{\prime}$ since $\left|C^{\prime}\right| \geq 3$. By the minimality of the gap $\left|C^{\prime}\right|+1$, we have

$$
d\left(x_{2},\left\{u_{1}, u_{3}\right\}\right)=d\left(u_{2},\left\{y_{1}, y_{C}^{-}\right\}\right)=0 .
$$

Therefore, $d\left(x_{2}\right) \leq 2 a-4$. From the minimality of the gap $\left|C^{\prime}\right|+1$ and $P_{1}$ it follows that $x_{1} u_{3} \notin A(D)$. Therefore, by $(2), d\left(u_{3}\right) \leq 2 a-3$. This together with condition $B_{0}$ and $\left\{y_{C}^{-}, u_{3}\right\} \rightarrow y$ imply that $d\left(y_{C}^{-}\right) \geq 2 a-2$. Therefore, by (2), the vertex $y_{C}^{-}$and every vertex of $X \backslash\left\{u_{2}\right\}$ form a 2-cycle, i.e., $y_{C}^{-} \leftrightarrow X \backslash\left\{u_{2}\right\}$. In particular, $y_{C}^{-} \leftrightarrow\left\{x_{2}, z\right\}$, where $z$ is an arbitrary vertex of $X \cap R \backslash\left\{u_{2}\right\}$. By the minimality of the gap, we have $a\left(z, u_{3}\right)=d^{-}\left(z,\left\{u_{1}\right\}\right)=0$. Therefore, $d(z) \leq 2 a-3$. This and $d\left(x_{2}\right) \leq 2 a-4$ contradict condition $B_{0}$ since $\left\{z, x_{2}\right\} \rightarrow y_{C}^{-}$.

Now consider the case $s=2$, i.e., $P_{1}=u_{1} u_{2}$. Notice that $u_{1}, y \in Y$ and $y_{C}^{-} \in X$ since $x_{1} \in X$. It is easy to see that $\left|C^{\prime}\right|$ is even.

Assume first that $\left|C^{\prime}\right| \geq 4$. By the minimality of the gap $\left|C^{\prime}\right|+1$,

$$
d\left(u_{2},\left\{y_{1}, y_{C}^{--}\right\}\right)=a\left(y_{C}^{-}, u_{1}\right)=0,
$$


where $y_{C}^{--}$denotes the predecessor of $y_{C}^{-}$on $C$. Therefore, $d\left(u_{2}\right) \leq 2 a-4$ and $d\left(y_{C}^{-}\right) \leq 2 a-2$. Since $\left\{u_{2}, y_{C}^{-}\right\} \rightarrow y$, i.e., $\left\{u_{2}, y_{C}^{-}\right\}$is a dominating pair, by condition $B_{0}$ we have, $d\left(y_{C}^{-}\right)=2 a-2$. This together with the second equality of (3) imply that $y_{C}^{-}$and every vertex of $Y \backslash\left\{u_{1}\right\}$ form a 2-cycle. In particular, $y \rightarrow y_{C}^{-} \rightarrow y_{1}$ and $y_{C}^{-} \leftrightarrow z$, where $z$ is an arbitrary vertex of $Y \cap R \backslash\left\{u_{1}\right\}$. From the minimality of the gap $\left|C^{\prime}\right|+1$ it follows that $a\left(z, u_{2}\right)=d^{-}\left(z,\left\{x_{2}\right\}\right)=0$. Hence, $d(z) \leq 2 a-3$. Now we consider the vertex $y_{C}^{--}$. It is easy to see that $y_{C}^{--} y_{C}^{+} \notin A(D)$ (for otherwise, the cycle $x_{1} u_{1} u_{2} y y_{C}^{-} y_{1} \ldots y_{C}^{--} y_{C}^{+} \ldots x_{1}$ is longer than $C$ ). From this and the first equality of (3) it follows that $d\left(y_{C}^{--}\right) \leq 2 a-3$. Thus, we have $\left\{z, y_{C}^{-}\right\} \rightarrow y_{C}^{-}$and $\max \left\{d(z), d\left(y_{C}^{--}\right)\right\} \leq 2 a-3$, which contradict condition $B_{0}$.

Assume then that $\left|C^{\prime}\right|=2$, i.e., $C^{\prime}=\left\{y_{1}, x_{2}\right\}$. Then $y=y_{2}$. By the minimality of the gap $\left|C^{\prime}\right|+1$,

$$
a\left(u_{1}, x_{2}\right)=a\left(u_{2}, y_{1}\right)=0
$$

i.e., the vertices $u_{1}$ and $x_{2}$ (respectively, $u_{2}$ and $y_{1}$ ) are not adjacent. Therefore,

$$
\max \left\{d\left(u_{1}\right), d\left(x_{2}\right), d\left(u_{2}\right), d\left(y_{1}\right)\right\} \leq 2 a-2 .
$$

Without loss of generality, we may assume that $d\left(x_{2}\right)=2 a-2$, since $\left\{u_{2}, x_{2}\right\} \rightarrow y_{2}$ (for otherwise, $d\left(u_{2}\right)=2 a-2$ and we will consider the cycle $\left.x_{1} u_{1} u_{2} y_{2} \ldots y_{m} x_{1}\right)$. Since $u_{1}$ and $x_{2}$ are non-adjacent and $d\left(x_{2}\right)=2 a-2$, it follows that $x_{2}$ and every vertex of $Y \backslash\left\{u_{1}\right\}$ form a 2-cycle, i.e., $x_{2} \leftrightarrow Y \backslash\left\{u_{1}\right\}$. In particular, $y_{2} \rightarrow x_{2} \rightarrow y_{1}$. Let $z$ be an arbitrary vertex in $Y \cap R \backslash\left\{u_{1}\right\}$. By the minimality of the gap $\left|C^{\prime}\right|+1, a\left(z, u_{2}\right)=0$ and $x_{1} z \notin A(D)$. Hence, $d(z) \leq 2 a-3$. If $y_{1} \rightarrow x_{3}$ (possibly, $x_{3}=x_{1}$ ), then, since $y_{2} \rightarrow x_{2} \rightarrow y_{1}$, we see that $x_{1} u_{1} u_{2} y_{2} x_{2} y_{1} x_{3} \ldots x_{1}$ is a cycle of length $|C|+2$, a contradiction. We may therefore assume that $y_{1} x_{3} \notin A(D)$. This together with $a\left(y_{1}, u_{2}\right)=0$ (by (4)) gives $d\left(y_{1}\right) \leq 2 a-3$. Thus we have that $\left\{y_{1}, z\right\} \rightarrow x_{2}$ and $\max \left\{d\left(y_{1}\right), d(z)\right\} \leq 2 a-3$, which contradict condition $B_{0}$. This contradiction completes the proof of $s=1$.

From $s=1$ it follows that $u_{1} \in Y$ and $y \in X$ since $x_{1} \in X$. Without loss of generality, we may assume that $y:=x_{r}$. From now on, let $y:=u_{1}$.

Now we divide the proof of the theorem into two parts: $\left|C^{\prime}\right|=1$ and $\left|C^{\prime}\right| \geq 2$.

Part I. $\left|C^{\prime}\right|=1$, i.e., $r=2$ and $x_{1} \rightarrow y \rightarrow x_{2}$.

By condition $B_{0}, \max \left\{d(y), d\left(y_{1}\right)\right\} \geq 2 a-2$ since $\left\{y, y_{1}\right\} \rightarrow x_{2}$. Without loss of generality, assume that $d(y) \geq 2 a-2$. For this part we first prove Claims 1-5 below.

Claim 1. If $x \in R \cap X$ and $x \leftrightarrow y$, then $d(x) \leq 2 a-3$ and $d\left(x_{1}\right) \geq 2 a-2$.

Proof of Claim 1. Assume that $x \in R \cap X$ and $x \leftrightarrow y$, but $d(x) \geq 2 a-2$. It is easy to see that $a\left(x, y_{1}\right)=0$ since $C$ is a longest non-Hamiltonian cycle in $D$. This and $d(x) \geq 2 a-2$ imply that the vertex $x$ and every vertex of $Y \backslash\left\{y_{1}\right\}$ form a 2-cycle. In particular, $x \leftrightarrow\left\{y_{0}, y_{2}, y_{m}\right\}$ (possibly, $y_{2}=y_{m}$ ), where $y_{0}$ is an arbitrary vertex of $Y \cap R \backslash\{y\}$. Using this, it is easy to check that

$$
a\left(x_{1}, y_{0}\right)=d^{-}\left(x_{1},\{y\}\right)=d^{-}\left(y,\left\{x_{2}\right\}\right)=0
$$

since $C$ is a longest non-Hamiltonian cycle in $D$. From the last equalities we have $d\left(x_{1}\right) \leq 2 a-3$, and $y \leftrightarrow x_{0}$, where $x_{0}$ is an arbitrary vertex of $X \cap R \backslash\{x\}$, since $d(y) \geq 2 a-2$. Since $C$ is a longest non-Hamiltonian cycle in $D$ and since $x \leftrightarrow\left\{y_{0}, y_{2}, y_{m}\right\}$, it follows that $d\left(x_{0},\left\{y_{1}, y_{0}\right\}\right)=0$. Therefore, $d\left(x_{0}\right) \leq 2 a-4$, which contradicts condition $B_{0}$ since $\max \left\{d\left(x_{1}\right), d\left(x_{0}\right)\right\} \leq 2 a-3$ and $\left\{x_{0}, x_{1}\right\} \rightarrow y$. This contradiction proves that $d(x) \leq 2 a-3$. From this and condition $B_{0}$ it follows that $d\left(x_{1}\right) \geq 2 a-2$ since $\left\{x, x_{1}\right\} \rightarrow y$. Claim 1 is proved. 
Claim 2 follows immediately from Claim 1 and condition $B_{0}$.

Claim 2. There are no two distinct vertices $x, x_{0} \in R \cap X$ such that $x \leftrightarrow y$ and $x_{0} \leftrightarrow y$.

Claim 3. If $y \leftrightarrow x$ for some $x \in R \cap X$, then $a(y, z)=1$ for all $z \in R \cap X \backslash\{x\}$.

Proof of Claim 3. Let $x \leftrightarrow y$ for some $x \in R \cap X$. Then Claim 2 implies that $a(y, z) \leq 1$ for all $z \in R \cap X \backslash\{x\}$.

We want to show that $a(y, z)=1$ for all $z \in R \cap X \backslash\{x\}$. Assume that this is not the case. Then $a\left(y, x_{0}\right)=0$ for some $x_{0} \in R \cap X \backslash\{x\}$. This together with Claims 1 and 2 thus imply that $|R|=4$ since $d(y) \geq 2 a-2$ by our assumption. Then $R \cap X=\left\{x, x_{0}\right\}$. Let $R \cap Y=\left\{y, y_{0}\right\}$. Since $a\left(y, x_{0}\right)=0$ and $d(y) \geq 2 a-2$, it follows that

$$
y \leftrightarrow\left\{x_{1}, x_{2}, \ldots, x_{m}\right\}
$$

Since $C$ is a longest non-Hamiltonian cycle in $D,(5)$ implies that $d\left(x,\left\{y_{1}, y_{2}, \ldots, y_{m}\right\}\right)=0$, which in turn implies that the vertices $x$ and $y_{0}$ are adjacent and $d(x) \leq 2 a-4$ since $U G(D)$ is 2-connected and $m \geq 2$. By the assumption of Claim 3 and (5), $\left\{x, x_{i}\right\} \rightarrow y$ for all $i \in[1, m]$. Now using condition $B_{0}$, we obtain

$$
d\left(x_{i}\right) \geq 2 a-2, \quad \text { for all } i \in[1, m] .
$$

The remainder of the proof of Claim 3 is divided into two subcases depending on the value of $a\left(x, y_{0}\right)$.

Case 1. $a\left(x, y_{0}\right)=2$, i.e., $x \leftrightarrow y_{0}$.

Since $C$ is a longest non-Hamiltonian cycle in $D$, from (5) and $y \leftrightarrow x, x \leftrightarrow y_{0}$ it follows that

$$
d\left(y_{0},\left\{x_{1}, x_{2}, \ldots, x_{m}\right\}\right)=0 .
$$

Therefore, since $U G(D)$ is 2-connected, the vertices $y_{0}$ and $x_{0}$ are adjacent. Using (5), it is not difficult to see that:

If $x_{0} \rightarrow y_{0}$, then $d^{-}\left(x_{0},\left\{y_{1}, y_{2}, \ldots, y_{m}\right\}\right)=0$, and if $y_{0} \rightarrow x_{0}$, then $d^{+}\left(x_{0},\left\{y_{1}, y_{2}, \ldots, y_{m}\right\}\right)=0$.

In both cases we have $a\left(y_{i}, x_{0}\right) \leq 1$ for all $i \in[1, m]$. Together with $a\left(y_{i}, x\right)=0$ this implies that $d\left(y_{i}\right) \leq 2 a-3$. On the other hand, from (6) and (7) it follows that every vertex $x_{i}, i \in[1, m]$, and every vertex of $Y \backslash\left\{y_{0}\right\}$ form a 2-cycle. In particular, $\left\{y_{1}, y_{2}\right\} \rightarrow x_{2}$, which contradicts condition $B_{0}$, since $\max \left\{d\left(y_{1}\right), d\left(y_{2}\right)\right\} \leq 2 a-3$.

Case 2. $a\left(x, y_{0}\right)=1$, i.e., $y_{0} \mapsto x$ or $x \mapsto y_{0}$.

Let $y_{0} \mapsto x$. Since $C$ is a longest non-Hamiltonian cycle in $D$ and (5), we have

$$
d^{-}\left(y_{0},\left\{x_{1}, x_{2}, \ldots, x_{m}\right\}\right)=0
$$

Hence $x_{0} \rightarrow y_{0}$ since $D$ is strong and $y_{0} \mapsto x$. Now using (5), we obtain

$$
d^{-}\left(x_{0},\left\{y_{1}, y_{2}, \ldots, y_{m}\right\}\right)=0
$$

The last two equalities together with $a\left(y, x_{0}\right)=0$ and $y_{0} \mapsto x$ imply that

$$
A\left(V(C) \cup\{x, y\} \rightarrow\left\{x_{0}, y_{0}\right\}\right)=\emptyset,
$$

which contradicts that $D$ is strong.

Let now $x \mapsto y_{0}$. Again using (5), it is easy to see that $d^{+}\left(y_{0},\left\{x, x_{1}, x_{2}, \ldots, x_{m}\right\}\right)=0$. Therefore, $y_{0} \rightarrow x_{0}$ since $D$ is strong. Together with (5) this implies that $d^{+}\left(x_{0},\left\{y_{1}, y_{2}, \ldots, y_{m}, y\right\}\right)=0$. Therefore $x_{0} \rightarrow y_{0}$ since $D$ is strong, and $d\left(x_{0}\right) \leq 2 a-4$ since $m \geq 2$. Thus, $d(x) \leq 2 a-3$ (Claim 1) and $d\left(x_{0}\right) \leq 2 a-4$, which contradicts condition $B_{0}$ since $\left\{x, x_{0}\right\} \rightarrow y_{0}$. This contradiction completes the proof of Claim 3 . 
Claim 4. If $y \leftrightarrow x$ for some $x \in R \cap X$, then $d^{-}(y, R \cap X \backslash\{x\})=0$.

Proof of Claim 4. Assume that the claim is not true, i.e., there exist vertices $x \in R \cap X$ and

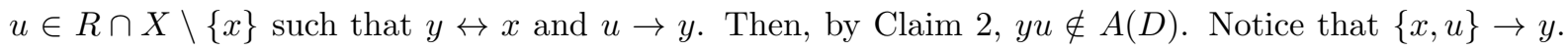
Since $d(x) \leq 2 a-3$ (Claim 1), condition $B_{0}$ implies that $d(u) \geq 2 a-2$. It is clear that $y_{1} u \notin A(D)$ (if $y_{1} \rightarrow u$, then $x_{1} y_{1} u y x_{2} y_{3} \ldots y_{m} x_{1}$ is a cycle of length $2 m+2$, a contradiction). Using this, $y u \notin A(D)$ and $d(u) \geq 2 a-2$ we conclude that $u y_{1} \in A(D), d(u)=2 a-2$ and the vertex $u$ together with every vertex of $Y \backslash\left\{y, y_{1}\right\}$ forms a 2-cycle. In particular, $u \leftrightarrow\left\{y_{2}, y_{m}, v\right\}$, where $v \in Y \cap R \backslash\{y\}$ (possibly, $\left.y_{2}=y_{m}\right)$. Using this, it is not difficult to show that $a\left(x_{1}, v\right)=0$ and $y x_{1} \notin A(D)$. Indeed, if $x_{1} \rightarrow v$, then $x_{1} v u y_{1} x_{2} \ldots y_{m} x_{1}$ is a cycle of length $2 m+2$; if $v \rightarrow x_{1}$, then $y_{m} u v x_{1} y_{1} \ldots x_{m} y_{m}$ is a cycle of length $2 m+2$; if $y \rightarrow x_{1}$, then $y_{m} u y x_{1} y_{1} \ldots x_{m} y_{m}$ is a cycle of length $2 m+2$. In each case we obtain a contradiction. Hence, $a\left(x_{1}, v\right)=0$ and $y x_{1} \notin A(D)$. From this it follows that $d\left(x_{1}\right) \leq 2 a-3$, which contradicts that $d\left(x_{1}\right) \geq 2 a-2$ (Claim 1$)$. Claim 4 is proved.

Claim 5. There is no $x \in X \cap R$ such that $y \leftrightarrow x$, i.e., in subdigraph $D\langle R\rangle$ through the vertex $y$ there is no cycle of length two.

Proof of Claim 5. Assume that the claim is not true, i.e., there exists a vertex $x \in X \cap R$ such that $y \leftrightarrow x$. By Claims 1, 3 and 4 we have

$$
d(x) \leq 2 a-3, \quad d\left(x_{1}\right) \geq 2 a-2 \quad \text { and } \quad y \mapsto X \cap R \backslash\{x\} .
$$

Case 1. $x_{2} y \notin D$.

Then from the last expression of (8) and $d(y) \geq 2 a-2$ we conclude that $|R|=4$, i.e., $m=a-2 \geq 3$, and the vertex $y$ and every vertex of $\left\{x_{1}, x_{2}, x_{3}, x_{4}, \ldots, x_{m}\right\} \backslash\left\{x_{2}\right\}$ form a 2-cycle, i.e.,

$$
y \leftrightarrow\left\{x_{1}, x_{3}, x_{4}, \ldots, x_{m}\right\} \quad \text { and } \quad y \mapsto x_{2} .
$$

Put $R=\left\{y, x, y_{0}, x_{0}\right\}$, where $x_{0} \in X$ and $y_{0} \in Y$. From (8) it follows that $y \mapsto x_{0}$. Now using (9), it is not difficult to see that

$$
d^{-}\left(x,\left\{y_{1}, y_{2}, \ldots, y_{m}\right\}\right)=d^{+}\left(x,\left\{y_{1}, y_{2}, \ldots, y_{m}\right\}-\left\{y_{2}\right\}\right)=0 .
$$

Indeed, if $y_{i} \rightarrow x$ and $i \in[1, m]$ (respectively, $x \rightarrow y_{j}$ and $j \in[1, m] \backslash\{2\}$ ), then $x_{i} y_{i} x y x_{i+1} \ldots x_{i}$ (respectively, $x_{j} y x y_{j} \ldots y_{j-1} x_{j}$ ) is a cycle of length $2 a-2$, a contradiction.

Similarly,

$$
d^{+}\left(x_{0},\left\{y_{1}, y_{2}, \ldots, y_{m}\right\}-\left\{y_{2}\right\}\right)=0 .
$$

In particular, (10) implies that

$$
d\left(x,\left\{y_{1}, y_{2}, \ldots, y_{m}\right\}-\left\{y_{2}\right\}\right)=0,
$$

i.e., the vertices $x$ and $y_{i}, i \notin\{0,2\}$, are not adjacent. From this, (11) and $y \mapsto x_{0}$ it follows that

$$
\max \left\{d\left(x_{0}\right), d(x), d\left(y_{j}\right)\right\} \leq 2 a-3, \quad \text { for all } j \notin\{0,2\} .
$$

Using (12) and condition $B_{0}$, we obtain that for all $u \in X$ and $v \in Y$ the following holds

$$
d^{-}\left(u,\left\{y_{1}, y_{m}\right\}\right) \leq 1 \quad \text { and } \quad d^{-}\left(v,\left\{x_{0}, x\right\}\right) \leq 1 .
$$

Now we divide this case into four subcases.

Subcase 1.1. $x \leftrightarrow y_{0}$.

Since $x \leftrightarrow y$ and $x \leftrightarrow y_{0}$, using (9) and the fact that $m \geq 3$, it is not difficult to check that the vertices $x_{1}$ and $y_{0}$ are not adjacent (for otherwise, $D$ would contain a cycle of length $2 a-2$, a contradiction). Together with the first inequality of (13) (when $u=x_{1}$ ) this implies that $d\left(x_{1}\right) \leq 2 a-3$, which contradicts Claim 1. 
Subcase 1.2. $x \mapsto y_{0}$.

Then, by the second inequality of (13) when $v=y_{0}, x_{0} y_{0} \notin A(D)$. This together with $x_{0} y \notin A(D)$ and (11) imply that $x_{0} \rightarrow y_{2}$ since $D$ is strong. It is easy to see that $y_{0} x_{2} \notin A(D)$ (for otherwise, $x_{1} y x y_{0} x_{2} y_{2} \ldots y_{m} x_{1}$ is a cycle of length $2 a-2$, a contradiction). Combining this with $x_{2} y \notin A(D)$ and $d^{-}\left(x_{2},\left\{y_{1}, y_{m}\right\}\right) \leq 1$ (by $\left.(13)\right)$ we obtain $d\left(x_{2}\right) \leq 2 a-3$. Thus, $d\left(x_{2}\right) \leq 2 a-3$ and $d\left(x_{0}\right) \leq 2 a-3$ (by (12)) and $\left\{x_{0}, x_{2}\right\} \rightarrow y_{2}$, which contradict condition $B_{0}$.

Subcase 1.3. $y_{0} \mapsto x$.

If $x_{i} \rightarrow y_{0}$ and $i \in[1, m]$, then using (9) we obtain that the cycle $x_{i} y_{0} x y x_{i+1} \ldots x_{i}$ has length $2 a-2$, which is a contradiction. We may therefore assume that $d^{-}\left(y_{0},\left\{x, x_{1}, x_{2}, \ldots, x_{m}\right\}\right)=0$. Hence, $x_{0} \rightarrow y_{0}$ since $D$ is strong and $y_{0} \mapsto x$. Since $x_{m} \rightarrow y \rightarrow x_{2}$ and $C$ is a longest non-Hamiltonian cycle in $D$, it follows that the vertices $x_{1}$ and $y_{0}$ are not adjacent. Combing this with $d^{-}\left(x_{1},\left\{y_{1}, y_{m}\right\}\right) \leq 1$ (by (13)) we obtain that $d\left(x_{1}\right) \leq 2 a-3$, a contradiction to Claim 1 .

Subcase 1.4. The vertices $x$ and $y_{0}$ are not adjacent.

Since the underlying undirected graph of $D$ is 2-connected, from $a\left(x, y_{0}\right)=0$ and (10) it follows that $x \rightarrow y_{2}$. Together with (13) this imply that $x_{0} y_{2} \notin A(D)$. Therefore, by (11), we have that $d^{+}\left(x_{0},\left\{y, y_{1}, y_{2}, \ldots, y_{m}\right\}\right)=0$. Hence, $x_{0} \rightarrow y_{0}$ since $D$ is strong. If $y_{0} \rightarrow x_{2}$, then $x_{1} y x_{0} y_{0} x_{2} y_{2} \ldots y_{m} x_{1}$ is a cycle of length $2 a-2$, which is a contradiction. We may therefore assume that $y_{0} x_{2} \notin A(D)$. Combining this with $x_{2} y \notin A(D)$ and (13) we obtain that $d\left(x_{2}\right) \leq 2 a-3$, which contradicts condition $B_{0}$ since $\left\{x_{2}, x\right\} \rightarrow y_{2}$ and $d(x) \leq 2 a-3$ (Claim 1). The discussion of Case 1 is completed.

Case 2. $x_{2} \rightarrow y$.

Since $y \mapsto X \cap R \backslash\{x\}$ (by (8)) and $d(y) \geq 2 a-2$, it follows that the vertices $y$ and $x_{i}$, where $i \in[1, m]$, are adjacent and $y \rightarrow\left\{x_{1}, x_{2}, \ldots, x_{m}\right\}$ or $\left\{x_{1}, x_{2}, \ldots, x_{m}\right\} \rightarrow y$. Therefore, without loss of generality, we may assume that

$$
x_{1} \rightarrow y \quad \text { and } \quad x_{i} \leftrightarrow y \quad \text { for all } i \in[2, m]
$$

(for otherwise we will have the considered Case 1). Let $x_{0}$ be an arbitrary vertex in $X \cap R \backslash\{x\}$. Then by (8) we have $y \mapsto x_{0}$. It is clear that

$$
d^{+}\left(x_{0},\left\{y, y_{1}, y_{2}, \ldots, y_{m}\right\}\right)=d\left(x,\left\{y_{1}, y_{2}, \ldots, y_{m-1}\right\}\right)=0 \quad \text { and } \quad x y_{m} \notin A(D)
$$

This implies that $d\left(x_{0}\right) \leq 2 a-3$. Since $D$ is strong and (15), there is a vertex $y_{0} \in Y \cap R \backslash\{y\}$ such that $x_{0} \rightarrow y_{0}$. Now, since $y \rightarrow x_{0} \rightarrow y_{0}$ and $x_{i} \rightarrow y$ for all $i \in[1, m]$ (by (14)), we conclude that $d^{+}\left(y_{0},\left\{x_{1}, x_{2}, \ldots, x_{m}\right\}\right)=0$ (for otherwise, for some $i \in[1, m], y_{0} \rightarrow x_{i}$ and $x_{i-1} y x_{0} y_{0} x_{i} \ldots y_{i-2} x_{i-1}$ is a cycle of length $2 m+2$, a contradiction). The last equality together with $d^{+}\left(x_{0},\left\{y, y_{1}, y_{2}, \ldots, y_{m}\right\}\right)=0$ (by (15)) imply that $y_{0} \rightarrow x$ since $D$ is strong (for otherwise, $A\left(\left\{x_{0}, y_{0}\right\} \rightarrow V(D) \backslash\left\{x_{0}, y_{0}\right\}\right)=\emptyset$, which contradicts that $D$ is strong). Then using the facts that $y \rightarrow x_{0} \rightarrow y_{0}$ and $y_{0} \rightarrow x \rightarrow y$, it is not difficult to show that $x_{1}$ and $y_{0}$ are not adjacent. Indeed, by (14) we have that: If $x_{1} \rightarrow y_{0}$, then $x_{1} y_{0} x y x_{2} y_{2} \ldots y_{m} x_{1}$ is a cycle of length $2 m+2$; and if $y_{0} \rightarrow x_{1}$, then $x_{m} y x_{0} y_{0} x_{1} y_{1} \ldots y_{m-1} x_{m}$ is a cycle of length $2 m+2$, in both cases we have a contradiction. So, $x_{1}$ and $y_{0}$ are not adjacent. Together with $d\left(x_{1}\right) \geq 2 a-2($ Claim 1 ) this implies that $y_{1} \rightarrow x_{1}$. On the other hand, since $d\left(x,\left\{y_{1}, y_{m}\right\}\right)=d^{+}\left(x_{0},\left\{y_{1}, y_{m}\right\}\right)=0$, we have that $\max \left\{d\left(y_{1}\right), d\left(y_{m}\right)\right\} \leq 2 a-3$, which contradicts condition $B_{0}$, because of $\left\{y_{m}, y_{1}\right\} \rightarrow x_{1}$. Claim 5 is proved.

Now we can finish the discussion of Part I.

From Claim 5 it follows that in $D\langle R\rangle$ there is no cycle of length two through the vertex $y$. Then, since $d(y) \geq 2 a-2$ and $|R| \geq 4$, it follows that $|R|=4$. Put $X \cap R=\left\{x, x_{0}\right\}$ and $Y \cap R=\left\{y, y_{0}\right\}$. Then 
$a(y, x)=a\left(y, x_{0}\right)=1$ and the vertex $y$ and every vertex of $X \cap C$ form a 2-cycle, i.e.,

$$
y \leftrightarrow\left\{x_{1}, x_{2}, \ldots, x_{m}\right\} .
$$

First consider the case $d^{+}\left(y,\left\{x, x_{0}\right\}\right) \geq 1$. Assume, without loss of generality, that $y \mapsto x$. Then, by (16), $d^{+}\left(x,\left\{y_{1}, y_{2}, \ldots, y_{m}\right\}\right)=0$. Together with $x y \notin A(D)$ this implies that $x \rightarrow y_{0}$ since $D$ is strong. Therefore $d(x) \leq 2 a-3$ since $|Y \cap C| \geq 2$. By (16), it is clear that $d^{+}\left(y_{0},\left\{x_{1}, x_{2}, \ldots, x_{m}\right\}\right)=0$. If $y \rightarrow x_{0}$, then analogously we obtain that $d^{+}\left(x_{0},\left\{y, y_{1}, y_{2}, \ldots, y_{m}\right\}\right)=0$ and $x_{0} \rightarrow y_{0}, d\left(x_{0}\right) \leq 2 a-3$, which contradicts condition $B_{0}$ since $\max \left\{d(x), d\left(x_{0}\right)\right\} \leq 2 a-3$ and $\left\{x, x_{0}\right\} \rightarrow y_{0}$. We may assume therefore that $y x_{0} \notin A(D)$. Then $x_{0} \rightarrow y$ (by $\left.a\left(y, x_{0}\right)=1\right), d^{-}\left(x_{0},\left\{y, y_{1}, y_{2}, \ldots, y_{m}\right\}\right)=0$ (by (16)) and hence, $y_{0} \rightarrow x_{0}$ since $D$ is strong. Now it is not difficult to show that

$$
d^{-}\left(y_{0},\left\{x_{1}, x_{2}, \ldots, x_{m}\right\}\right)=d^{-}\left(x_{0},\left\{y_{1}, y_{2}, \ldots, y_{m}\right\}\right)=d^{+}\left(x,\left\{y_{1}, y_{2}, \ldots, y_{m}\right\}\right)=0 .
$$

Therefore $d\left(x_{0}\right) \leq 2 a-3$ and $d\left(y_{i}\right) \leq 2 a-3$. Since for all $i \in[1, m],\left\{x_{i}, x_{0}\right\} \rightarrow y$ and $d\left(x_{0}\right) \leq 2 a-3$, from condition $B_{0}$ it follows that $d\left(x_{i}\right) \geq 2 a-2$ for all $i \in[1, m]$. This together with $a\left(x_{i}, y_{0}\right)=0$ (by (16) and the last equalities) imply that $y_{i} \leftrightarrow x_{i}$. Thus, $\left\{y_{i-1}, y_{i}\right\} \rightarrow x_{i}$ and $\max \left\{d\left(y_{i-1}\right), d\left(y_{i}\right)\right\} \leq 2 a-3$, which is a contradiction.

Now consider the case $d^{+}\left(y,\left\{x, x_{0}\right\}\right)=0$. Then $\left\{x, x_{0}\right\} \rightarrow y$, because of $a(y, x)=a\left(y, x_{0}\right)=1$, i.e., $\left\{x, x_{0}\right\}$ is a dominating pair. It is clear that $d^{-}\left(x,\left\{y_{1}, y_{2}\right\}\right)=d^{-}\left(x_{0},\left\{y_{1}, y_{2}\right\}\right)=0$. This together with $d^{+}\left(y,\left\{x_{0}, x\right\}\right)=0$ imply that $\max \left\{d(x), d\left(x_{0}\right)\right\} \leq 2 a-3$, which is a contradiction because of $\left\{x, x_{0}\right\} \rightarrow y$. This completes the discussion of the part $\left|C^{\prime}\right|=1$.

Part 2. $\left|C^{\prime}\right| \geq 2$.

Then $\left|C^{\prime}\right| \geq 3$ since $\left|C^{\prime}\right|$ is odd. For this part we first will prove Claims 6-8.

Claim 6. If $\left|C^{\prime}\right| \geq 3$, then the following holds:

(a). $d(y) \leq 2 a-3$ and $d\left(y_{r-1}\right) \geq 2 a-2$;

(b). There is no $x \in X \cap R$ such that $x \leftrightarrow y_{r-1}$, i.e., $a\left(y_{r-1}, x\right) \leq 1$ for all $x \in X \cap R$;

(c). $a\left(y_{r-1}, x\right)=1$ for all $x \in X \cap R,|R|=4, d\left(y_{r-1}\right)=2 a-2$ and the vertex $y_{r-1}$ together with every vertex of $X \cap V(C)$ forms a 2-cycle. In particular, $x_{r} \leftrightarrow y_{r-1}$ and $y_{r-1} \leftrightarrow x_{2}$;

(d). $d^{-}\left(y_{r-1},\left\{x, x_{0}\right\}\right)=0$ and $y_{r-1} \mapsto\left\{x, x_{0}\right\}$;

(e). $\max \left\{d\left(x_{0}\right), d(x)\right\} \leq 2 a-3$ and $d^{-}\left(v,\left\{x_{0}, x\right\}\right) \leq 1$ for all $v \in Y$;

(f). $\left|C^{\prime}\right|=3$, i.e., $r=3$.

\section{Proof of Claim 6.}

(a). Suppose on the contrary, that $d(y) \geq 2 a-2$. Then, since $d\left(y, C^{\prime}\right)=0$, we have that $r=3$ and the vertex $y$ together with every vertex of $X \backslash\left\{x_{2}\right\}$ forms a 2-cycle. In particular, $y \leftrightarrow x_{1}$ and $y \leftrightarrow x_{3}$. It follows that for some $i \in[3, m], x_{i} \rightarrow y \rightarrow x_{i+1}$, which contradicts that $C$-bypass $P$ has the minimum gap among the gaps of all $C$-bypasses. Therefore, $d(y) \leq 2 a-3$. Now, since $\left\{y, y_{r-1}\right\} \rightarrow x_{r}$, from condition $B_{0}$ it follows that $d\left(y_{r-1}\right) \geq 2 a-2$.

(b). Suppose that Claim 6(b) is falls, i.e., there is a vertex $x \in X \cap R$ such that $x \leftrightarrow y_{r-1}$. From the minimality of the gap $\left|C^{\prime}\right|+1$ it follows that

$$
a(x, y)=d^{-}\left(x,\left\{y_{1}, y_{2}, \ldots, y_{r-2}\right\}\right)=0 \quad \text { and } \quad x y_{r} \notin A(D) .
$$

From this we have $d(x) \leq 2 a-4$. This together with condition $B_{0}$ and $\left\{x, x_{r-1}\right\} \rightarrow y_{r-1}$ imply that $d\left(x_{r-1}\right) \geq 2 a-2$. Therefore, since $a\left(x_{r-1}, y\right)=0$, it follows that

(i) the vertex $x_{r-1}$ and every vertex of $Y \backslash\{y\}$ form a 2-cycle. 
Let $y^{\prime}$ be an arbitrary vertex in $Y \cap R \backslash\{y\}$. By (i), $x_{r-1} \leftrightarrow y^{\prime}$. By the minimality of the gap $\left|C^{\prime}\right|+1$, $d^{+}\left(y^{\prime},\left\{x, x_{r}\right\}\right)=d^{-}\left(y^{\prime},\left\{x_{1}\right\}\right)=0$. Therefore, $d\left(y^{\prime}\right) \leq 2 a-3$. Since $\left\{y^{\prime}, y_{r-2}\right\} \rightarrow x_{r-1}$, condition $B_{0}$ implies that

$$
d\left(y_{r-2}\right) \geq 2 a-2 .
$$

First consider the case $r \geq 4$. Then $x_{r-2} \in C^{\prime}$ and it is not difficult to see that $x_{r-2} y^{\prime} \notin A(D)$ and $y_{r-2} x \notin A(D)$. Now, since $a\left(x_{r-2}, y\right)=0$, we have $d\left(x_{r-2}\right) \leq 2 a-3$. Using condition $B_{0}$ and the fact that $d(x) \leq 2 a-4$, we conclude that $x y_{r-2} \notin A(D)$. Therefore, by (17), $x$ and $y_{r-2}$ are not adjacent. This together with $d\left(y_{r-2}\right) \geq 2 a-2$ (by (18)) imply that $y_{r-2} \leftrightarrow x_{0}$, where $x_{0}$ is an arbitrary vertex in $R \cap X \backslash\{x\}$. By the minimality of the gap $\left|C^{\prime}\right|+1$, we have $x_{0} y_{r-1} \notin A(D), y_{r-3} x_{0} \notin A(D)$ and $x_{0} y^{\prime} \notin A(D)$. Therefore, $d\left(x_{0}\right) \leq 2 a-3$, which contradicts condition $B_{0}$, since $\left\{x_{0}, x_{r-2}\right\} \rightarrow y_{r-2}$ and $d\left(x_{r-2}\right) \leq 2 a-3$.

Now consider the case $r=3$. By (i), $x_{2} \leftrightarrow y_{3}$ and $x_{2} \leftrightarrow y_{2}$. If $y_{2} \rightarrow x_{4}$ (possibly, $x_{4}=x_{1}$ ) (respectively, $x_{3} \rightarrow y_{2}$ ), then the cycle $Q:=x_{1} y x_{3} y_{3} x_{2} y_{2} x_{4} \ldots y_{m} x_{1}$ (respectively, $Q:=x_{1} y x_{3} y_{2} x_{2} y_{3} x_{4} \ldots y_{m} x_{1}$ ) has length $2 m$, the vertex $y_{1}$ is not on this cycle and $x_{1} \rightarrow y_{1} \rightarrow x_{2}$ is a $Q$-bypass whose gap with respect to $Q$ is equal to 4 and $d\left(y_{1}\right) \geq 2 a-2$, (by (18) since $r-2=1$ ), which contradicts Claim 6(a). We may therefore assume that $y_{2} x_{4} \notin A(D)$ and $x_{3} y_{2} \notin A(D)$. Combining this with $d\left(y_{2}\right) \geq 2 a-2$ (by Claim 6 (a) and $r=3$ ) we obtain that $d\left(y_{2}\right)=2 a-2$ and $y_{2}$ together with every vertex of $X \backslash\left\{x_{3}, x_{4}\right\}$ forms a 2-cycle. In particular, $y_{2} \leftrightarrow x_{0}$. On the other hand, from the minimality of the gap $\left|C^{\prime}\right|+1$ it follows that $x_{0}, y$ are not adjacent and $y_{1} x_{0} \notin A(D)$. Therefore, $d\left(x_{0}\right) \leq 2 a-3$, which is a contradiction, since $d(x) \leq 2 a-3$ and $\left\{x, x_{0}\right\} \rightarrow y_{2}$.

(c). Claim 6(c) is an immediate corollary of Claims 6(a) and 6(b).

From now on, we assume that $X \cap R=\left\{x, x_{0}\right\}$ and $Y \cap R=\left\{y, y_{0}\right\}$.

(d). Suppose, on the contrary, that there exists a vertex in $\left\{x, x_{0}\right\}$, say $x$, such that $x \rightarrow y_{r-1}$. From the minimality of the gap $\left|C^{\prime}\right|+1$ it follows that $d^{-}\left(x,\left\{y, y_{1}\right\}\right)=0$. This together with $y_{r-1} x \notin A(D)$ (Claim 6(b)) imply that $d(x) \leq 2 a-3$. Therefore, using Claim 6(c) and condition $B_{0}$, we obtain that $d\left(x_{2}\right) \geq 2 a-2$ and $d\left(x_{r-1}\right) \geq 2 a-2$ (possibly, $\left.x_{2}=x_{r-1}\right)$ since $\left\{x, x_{2}, x_{r-1}\right\} \rightarrow y_{r-1}$. Together with $d\left(y,\left\{x_{2}, x_{r-1}\right\}\right)=0$ this implies that $x_{r-1} \rightarrow y_{r}$ and $x_{2} \leftrightarrow y_{0}$. Because of gap minimality, $d^{-}\left(y_{0},\left\{x_{1}\right\}\right)=d^{+}\left(y_{0},\left\{x, x_{r}\right\}\right)=0$. Therefore, $d\left(y_{0}\right) \leq 2 a-3$, and hence, by condition $B_{0}$, $d\left(y_{1}\right) \geq 2 a-2$ since $\left\{y_{1}, y_{0}\right\} \rightarrow x_{2}$. Since $x_{r} \rightarrow y_{r-1}$ and $y_{r-1} \rightarrow x_{2}$ (Claim 6(c)) and $x_{r-1} \rightarrow y_{r}$, the cycle $Q:=x_{1} y x_{r} y_{r-1} x_{2} \ldots x_{r-1} y_{r} \ldots y_{m} x_{1}$ has length equal to $2 a-4$. Observe that $y_{1} \notin V(Q)$ and $x_{1} \rightarrow y_{1} \rightarrow x_{2}$ is a $Q$-bypass, whose gap with respect to $Q$ is equal to 4 , but $d\left(y_{1}\right) \geq 2 a-2$, which contradicts Claim 6(a).

(e). By Claim 6(d), $y_{r-1} \mapsto\left\{x, x_{0}\right\}$. Therefore, because of gap minimality, we have

$$
A\left(\left\{x, x_{0}\right\} \rightarrow\left\{y, y_{r-1}, y_{r}\right\}\right)=\emptyset .
$$

Therefore, $\max \left\{d\left(x_{0}\right), d(x)\right\} \leq 2 a-3$ and, by condition $B_{0}$, the vertices $x, x_{0}$ does not form a dominating pair, i.e., $d^{-}\left(v,\left\{x_{0}, x\right\}\right) \leq 1$ for all $v \in Y$.

(f). Suppose, on the contrary, that is $\left|C^{\prime}\right|>3$. Then $\left|C^{\prime}\right| \geq 5$, i.e., $r \geq 4$, since $\left|C^{\prime}\right|$ is odd. By Claim 6(c), $x_{i} \leftrightarrow y_{r-1}$ for all $i \in[1, m]$. This together with condition $B_{0}$ imply that $d\left(x_{i}\right) \geq 2 a-2$ for all $i \in[1, m]$ maybe except one. In particular, $d\left(x_{r-1}\right) \geq 2 a-2$ or $d\left(x_{r-2}\right) \geq 2 a-2$.

Fist consider the case $d\left(x_{r-1}\right) \geq 2 a-2$. From this and $a\left(y, x_{r-1}\right)=0$ it follows that $x_{r-1} \leftrightarrow y_{r}$. Using this and the fact that $x_{r} \rightarrow y_{r-1} \rightarrow x_{2}$ (Claim 6(c)), we see that the cycle $Q:=x_{1} y x_{r} y_{r-1} x_{2} y_{2} \ldots x_{r-1} y_{r}$ 
$x_{r+1} \ldots x_{1}$ has length equal to $2 a-4$. Observe that $x_{1} \rightarrow y_{1} \rightarrow x_{2}$ is a $Q$-bypass whose gap with respect to $Q$ is equal to 4 , which contradicts the choice of the cycle $C$ and $C$-bypass $P$.

Now consider the case $d\left(x_{r-1}\right) \leq 2 a-3$. Then $d\left(x_{r-2}\right) \geq 2 a-2$. Observe that the vertex $x_{r-2}$ and every vertex of $Y$ other than $y$ form a 2-cycle (since $x_{r-2}$ and $y$ are not adjacent), in particular, $y_{0} \leftrightarrow x_{r-2}$. It is easy to see that $x_{r-2}=x_{2}$, i.e., $r=4$. Indeed, if $r-2 \geq 3$, then $x_{r-3} \in C^{\prime}$ and $x_{r-3} y_{0} \notin A(D)$ because of the minimality of the gap $\left|C^{\prime}\right|+1$ and $y_{0} \leftrightarrow x_{r-2}$. This together with $a\left(y, x_{r-3}\right)=0$ gives $d\left(x_{r-3}\right) \leq 2 a-3$. Thus we have that the vertices $x_{r-3}$ and $x_{r-1}$ both have degree less than $2 a-2$, which contradicts the fact that at most one vertex $x_{i}, i \in[1, m]$ maybe has degree less that $2 a-2$. Thus, $r=4$. By the above observations, $y_{4} \rightarrow x_{2}$ and $y_{3} \rightarrow x_{5}$. Therefore, $Q:=x_{1} y x_{4} y_{4} x_{2} y_{2} x_{3} y_{3} x_{5} \ldots x_{1}$ is a cycle of length $2 a-4$. Notice that $y_{1} \notin V(Q)$ and the $Q$-bypass $x_{1} \rightarrow y_{1} \rightarrow x_{2}$ has gap with respect to $Q$ equal to 4 . This contradicts the choice of $C$ and $C$-bypass $P$.

From Claims 6(c), 6(d) and 6(f)1 it follows that

(ii) If $\left|C^{\prime}\right| \geq 3$, then $\left|C^{\prime}\right|=3,|R|=4, y_{2} \mapsto\left\{x, x_{0}\right\}, d(y) \leq 2 a-3, d\left(y_{2}\right)=2 a-2$ and the vertex $y_{2}$ together with every vertex of $X \cap V(C)$ forms a 2-cycle.

Claim 7. If $\left|C^{\prime}\right|=3$, then $d\left(y_{0}\right) \leq 2 a-3$, (recall that $\left\{y_{0}\right\}=Y \cap R \backslash\{y\}$ ).

Proof of Claim 7. By Claim $6\left((\mathrm{e}), d^{-}\left(y_{0},\left\{x, x_{0}\right\}\right) \leq 1\right.$. Therefore, if $x_{2}$ and $y_{0}$ are not adjacent, then $d\left(y_{0}\right) \leq 2 a-3$. We may therefore assume that $x_{2}$ and $y_{0}$ are adjacent. Then $y_{0} \rightarrow x_{2}$ or $x_{2} \rightarrow y_{0}$. It is easy to see that if $y_{0} \rightarrow x_{2}$, then $x_{1} y_{0} \notin A(D)$; and if $x_{2} \rightarrow y_{0}$, then $y_{0} x_{3} \notin A(D)$. Therefore, $d\left(y_{0},\left\{x_{1}, x_{2}, x_{3}\right\}\right) \leq 4$. This and $d^{-}\left(y_{0},\left\{x_{0}, x\right\}\right) \leq 1$ imply that $d\left(y_{0}\right) \leq 2 a-3$.

Combining Claims 6(a), 6(e) and 7 we obtain that if $\left|C^{\prime}\right|=3$, then

$$
\max \left\{d(y), d\left(y_{0}\right), d(x), d\left(x_{0}\right)\right\} \leq 2 a-3,
$$

in particular, by condition $B_{0}$, we have

$$
\max \left\{d^{-}\left(u,\left\{y, y_{0}\right\}\right), d^{-}\left(v,\left\{x, x_{0}\right\}\right)\right\} \leq 1 \quad \text { for all } \quad u \in X \quad \text { and } \quad v \in Y \text {. }
$$

Claim 8. If $\left|C^{\prime}\right|=3$, then the following holds:

(a). $d\left(x_{2}\right) \leq 2 a-3$ and $d\left(x_{3}\right) \geq 2 a-2$;

(b). $d\left(y_{1}\right) \geq 2 a-2$;

(c). If $x \rightarrow y_{k}$ or $x_{0} \rightarrow y_{k}$, where $k \in[3, m]$, then $x_{k} y_{1} \notin A(D)$.

Proof of Claim 8.

(a). Suppose, on the contrary, that $\left|C^{\prime}\right|=3$ and $d\left(x_{2}\right) \geq 2 a-2$. This and $a\left(x_{2}, y\right)=0$ imply that $d\left(x_{2}\right)=2 a-2$ and $x_{2}$ together with every vertex of $Y \backslash\{y\}$ form a 2-cycle. In particular, $x_{2} \leftrightarrow y_{0}$ and $x_{2} \leftrightarrow y_{3}$. Since $d\left(y_{0}\right) \leq 2 a-3$ (by (19)) and $\left\{y_{1}, y_{0}\right\} \rightarrow x_{2}$, from condition $B_{0}$ it follows that $d\left(y_{1}\right) \geq 2 a-2$. On the other hand, using the facts that $x_{3} \rightarrow y_{2} \rightarrow x_{2}$ (Claim $\left.6(\mathrm{c})\right)$ and $x_{2} \rightarrow y_{3}$ we see that $Q:=x_{1} y x_{3} y_{2} x_{2} y_{3} \ldots x_{1}$ is a cycle of length $2 a-4$. Notice that $x_{1} \rightarrow y_{1} \rightarrow x_{2}$ is a $Q$-bypass, whose the gap with respect to $Q$ is equal to 4 . This contradicts the minimality of the gap $\left|C^{\prime}\right|+1$ or Claim 6(a) since $d\left(y_{1}\right) \geq 2 a-2$. Therefore, $d\left(x_{2}\right) \leq 2 a-3$. Together with condition $B_{0}$ this implies that $d\left(x_{3}\right) \geq 2 a-2$ since $\left\{x_{2}, x_{3}\right\} \rightarrow y_{2}$.

(b). Suppose, on the contrary, that $\left|C^{\prime}\right|=3$ and $d\left(y_{1}\right) \leq 2 a-3$. By condition $B_{0}, y_{1} x_{3} \notin A(D)$ since $d(y) \leq 2 a-3($ Claim $6(\mathrm{a}))$ and $y \rightarrow x_{3}$. From $(20)$ we have that $y_{0} x_{3} \notin A(D)$ since $y x_{3} \in A(D)$. Thus, the arcs $y_{1} x_{3}$ and $y_{0} x_{3}$ are not in $A(D)$. This together with $d\left(x_{3}\right) \geq 2 a-2$ (Claim $\left.8(\mathrm{a})\right)$ imply that $x_{3} \rightarrow y_{1}$ and $y_{3} \rightarrow x_{3}$. By (ii), $y_{2} \rightarrow x_{4}$ and hence, $Q:=x_{1} y x_{3} y_{1} x_{2} y_{2} x_{4} \ldots x_{1}$ is a cycle of length $2 a-4$, which does not contain the vertices $x_{0}, y_{0}, x$ and $y_{3}$. The path $x_{3} y_{3} x_{4}$ is a $Q$-bypass 
whose the gap with respect to $Q$ is equal to 4 . Therefore, by the minimality of the gap $\left|C^{\prime}\right|+1$ and Claim 6(a), $d\left(y_{3}\right) \leq 2 a-3$ but this is a contradiction since $\left\{y, y_{3}\right\} \rightarrow x_{3}$ and $d(y) \leq 2 a-3$ (Claim $6(\mathrm{a})$ ).

(c). Suppose that the claim is not true. Without loss of generality, assume that for some $k \in[3, m]$, $x \rightarrow y_{k}$ and $x_{k} \rightarrow y_{1}$. Then, by (ii) we have that $y_{2} \rightarrow\left\{x, x_{0}\right\}$ and hence, the cycle $x_{1} y x_{3} \ldots x_{k} y_{1} x_{2} y_{2} x y_{k}$ $\ldots y_{m} x_{1}$ is a cycle of length $2 a-2$, a contradiction.

Now we are ready to complete the proof of Theorem 1.7. Combining (19) and Claim 8(a), we obtain

$$
\max \left\{d(x), d\left(x_{0}\right), d(y), d\left(y_{0}\right), d\left(x_{2}\right)\right\} \leq 2 a-3 .
$$

This and condition $B_{0}$ imply

$$
d^{-}\left(v,\left\{x, x_{0}, x_{2}\right\}\right) \leq 1 \text { for all } v \in Y \text {. }
$$

Therefore, since $d\left(y_{1}\right) \geq 2 a-2$ (Claim $\left.8(\mathrm{~b})\right)$, the vertex $y_{1}$ and every vertex of $X \backslash\left\{x, x_{0}, x_{2}\right\}$ form a 2-cycle, i.e.,

$$
y_{1} \leftrightarrow x_{i} \quad \text { for all } \quad i \in[1, m] \backslash\{2\} .
$$

Now using Claim 8(c), we obtain $A\left(\left\{x, x_{0}\right\} \rightarrow\left\{y_{2}, y_{3}, \ldots, y_{m}\right\}\right)=\emptyset$. From $y_{2} \mapsto\left\{x, x_{0}\right\}$ (Claim 6(d)) and the minimality of the gap $\left|C^{\prime}\right|+1$ it follows that $d^{-}\left(y,\left\{x, x_{0}\right\}\right)=0$. The last two equalities imply that

$$
A\left(\left\{x, x_{0}\right\} \rightarrow\left\{y, y_{2}, y_{3}, \ldots, y_{m}\right\}\right)=\emptyset .
$$

By (20), in particular, we have

$$
\max \left\{d^{-}\left(y_{1},\left\{x, x_{0}\right\}\right), d^{-}\left(y_{0},\left\{x, x_{0}\right\}\right)\right\} \leq 1 .
$$

Since $D$ is strong, from (23) and (24) it follows that $x \rightarrow y_{0}$ or $x_{0} \rightarrow y_{0}$. Again using (24), we obtain that if $x \rightarrow y_{0}$, then $x_{0} y_{0} \notin A(D)$ and $x_{0} \rightarrow y_{1}$; if $x_{0} \rightarrow y_{0}$, then $x y_{0} \notin A(D)$ and $x \rightarrow y_{1}$.

Because of the symmetry between the vertices $x$ and $x_{0}$, we can assume that $x_{0} \rightarrow y_{0}, x \rightarrow y_{1}$ and $x_{0} y_{1} \notin A(D), x y_{0} \notin A(D)$. It is not difficult to show that $x_{2}$ and every vertex $y_{i}$ with $i \in[3, m]$ are not adjacent. Indeed, if $y_{i} \rightarrow x_{2}$, then, by (22), $y_{1} \rightarrow x_{i+1}$ and hence, $x_{1} y x_{3} y_{3} \ldots y_{i} x_{2} y_{2} x y_{1} x_{i+1} \ldots x_{1}$ is a cycle of length $2 a-2$; if $x_{2} \rightarrow y_{i}$, then, by (ii), $x_{i} \rightarrow y_{2}$ and hence, $x_{1} y x_{3} y_{3} \ldots x_{i} y_{2} x y_{1} x_{2} y_{i} \ldots y_{m} x_{1}$ is a cycle of length $2 a-2$. Thus, in both cases we have a contradiction.

Therefore, $a\left(y_{i}, x_{2}\right)=0$ for all $i \in[3, m]$. This and (20) imply that $d\left(y_{i}\right) \leq 2 a-3$ for all $i \in[3, m]$. From $y \rightarrow x_{3}, d(y) \leq 2 a-3$ (Claim $\left.6(\mathrm{a})\right)$ and condition $B_{0}$ it follows that $d^{-}\left(x_{3},\left\{y_{0}, y_{3}, y_{4}, \ldots y_{m}\right\}\right)=0$. Now frome $d\left(x_{3}\right) \geq 2 a-2$ (Claim $8\left(\right.$ a) ), we have that $m=3$, i.e., $a=5$. Since $x \rightarrow y_{1}, x_{0} \rightarrow y_{0}$ and (21), it follows that $d^{+}\left(x_{2},\left\{y_{0}, y_{1}\right\}\right)=0$. Because of $d\left(y_{1}\right) \geq 2 a-2$ (Claim 8(b)) and $d^{-}\left(y_{1},\left\{x_{0}, x_{2}\right\}\right)=0$ we have $y_{1} \rightarrow x_{0}, x_{3} \leftrightarrow y_{1}$ and $y_{1} \rightarrow x_{1}$. From this it follows that $y_{0} x_{2} \notin A(D)$ (for otherwise, $y_{1} x_{0} y_{0} x_{2} y_{2} \ldots x_{1} y_{1}$ is a cycle of length $2 a-2$, a contradiction). Therefore, $a\left(x_{2}, y_{0}\right)=0$. If $y_{0} \rightarrow x_{1}$, then the cycle $x_{1} y x_{3} y_{1} x_{2} y_{2} x_{0} y_{0} x_{1}$ is a cycle of length $2 a-2=8$, a contradiction. Therefore, $y_{0} x_{1} \notin A(D)$. So, we have $d^{+}\left(y_{0},\left\{x_{1}, x_{2}, x_{3}\right\}\right)=0$. Then $d^{+}\left(y_{0},\left\{x, x_{0}\right\}\right) \geq 1$ since $D$ is strong. It is easy to see that $y_{0} x \notin A(D)$ (for otherwise, $x_{1} y x_{3} y_{2} x_{0} y_{0} x y_{1} x_{1}$ is a cycle of length 8 , a contradiction. Therefore, $d^{+}\left(y_{0},\left\{x_{1}, x_{2}, x_{3}, x\right\}\right)=0$. On the other hand, from (23) and $x_{0} y_{1} \notin A(D)$ we have $N^{+}\left(x_{0}\right)=\left\{y_{0}\right\}$. Now it is not difficult to see that there is no path from $x_{0}$ to any vertex of $V(C)$ since $N^{+}\left(y_{0}\right)=\left\{x_{0}\right\}$, which contradicts that $D$ is strong. So, the discussion of the case $\left|C^{\prime}\right| \geq 3$ is completed. Theorem 1.7 is proved. 


\section{Concluding remarks}

In view of Theorem 1.3 it is natural to set the following problem.

Problem. Characterize those strongly connected balanced bipartite digraphs of order $2 a \geq 6$ in which $d(x)+d(y) \geq 3 a$ for every pair of vertices $x, y$ with a common in-neighbour or a common out-neighbour but are not even pancyclic.

\section{References}

[1] J. Adamus, A degree sum condition for hamiltonicity in balanced bipartite digraphs, arXiv: 1512.00480v1 [math.CO] 1 Dec 2015.

[2] J. Adamus, L. Adamus and A. Yeo, On the Meyniel condition for hamiltonicity in bipartite digraphs, Discrete Math. and Theoretical Comp. Science, 16(1) (2014), 293-302.

[3] J. Bang-Jensen, Y. Guo, A.Yeo, A new sufficient condition for a digraph to be Hamiltonian, Discrete Appl. Math., 95 (1999) 61-72.

[4] J. Bang-Jensen, G. Gutin, Digraphs: Theory, Algorithms and Applications, Springer, 2001.

[5] J. Bang-Jensen, G. Gutin, H. Li, Sufficient conditions for a digraph to be Hamiltonian, J. Graph Theory, 22(2) (1996) 181-187.

[6] L.W. Beineke, C. Little, Cycles in bipartite tournaments, J. Combin. Theory Ser. B, 32(2) (1982) 140-145.

[7] J.C. Bermond and C. Thomassen, Cycles in digraphs-a survey, J. Graph Theory 5 (1981), 1-43.

[8] J.A. Bondy, Basic graph theory: paths and circuits, in Handbook of combinatorics 1-2, Elsevier, Amsterdam, 1995.

[9] V. Chvátal, New directions in Hamiltonian graph theory , in "New directions in the theory of graphs" ( F. Harary, ed.), Academic Press, New York, (1973) 65-95.

[10] S.Kh. Darbinyan, Pancyclicity of digraphs with the Meyniel condition, Studia Scientiarum Mathematicarum Hungarica, 20(1-4) (1985) 95-117) Ph. D. Thesis, Institute Mathematici Akademy Nauk BSSR, Minsk, 1981 (in Russian).

[11] S.Kh. Darbinyan, A sufficient condition for the Hamiltonian property of digraphs with large semidegrees, Akademy Nauk Armyan. SSR Doklady, 82(1) (1986) 6-8 (see also arXiv: 1111.1843v1 [math.CO] 8 Nov 2011).

[12] S.Kh. Darbinyan, On the pancyclicity of digraphs with large semidegrees, Akademy Nauk Armyan. SSR Doklady, 83(3) (1986) 99-101 (see also arXiv: 1111.1841v1 [math.CO] 8 Nov 2011).

[13] S.Kh. Darbinyan, On pre-Hamiltonian cycles in balanced bipartite digraphs, Mathematical problems of computer science vol. 46 (2016) 7-17.

[14] S.Kh. Darbinyan, Sufficient conditions for Hamiltonian cycles in bipartite digraphs., (arXiv: 1604.08773v1 [math.CO] 29 Apr 2016).

[15] S.Kh. Darbinyan, Sufficient conditions for balanced bipartite digraphs to be even pancyclic., (arXiv: 1604.08773v1 [math.CO] 29 Apr 2016). 
[16] A. Ghouila-Houri, Une condition suffisante d'existence d'un circuit hamiltonien, Comptes Rendus de I' Academie des Sciences Paris Ser. A-B, 251 (1960) 495-497.

[17] G. Gutin, Cycles and paths in semicomplete multipartite digraphs, theorems and algorithms: a survey. J. Graph Theory 19 (4) (1995), 481-505.

[18] G. Gutin, A characterization of vertex pancyclic partly oriented $k$-partite tournaments, Vestsi Acad. Navuk BSSR Ser. Fiz.-Mat. Navuk 2 (1989) 41-46.

[19] G. Gutin, Characterizations of vertex pancyclic and pancyclic ordinary complete multipartite digraphs, Discrete Mathematics, 141 (1995) 153-162.

[20] R. Häggkvist, C. Thomassen, On pancyclic digraphs, J. Combin. Theory Ser. B, 20(1), (1976) 20-40.

[21] D. Kühn and D. Ostus, A survey on Hamilton cycles in directed graphs, European J. Combin. 33 (2012) 750-766.

[22] M. Meyniel, Une condition suffisante d'existence d'un circuit hamiltonien dans un graphe oriente, J. Combin. Theory Ser. B, 14 (1973) 137-147.

[23] C.St.J.A. Nash-Williams, Hamilton circuits in graphs and digraphs, in " The many facets of graph theory", Springer, Lecture Notes 110 (1969), 237-243.

[24] C. Thomassen, An Ore-type condition implying a digraph to be pancyclic, Discrete Math., 19(1) (1977) 85-92.

[25] C. Thomassen, Long cycles in digraphs, Proc. Lond. Math. Soc., 3(42) (1981) 231-251.

[26] R. Wang, A sufficient condition for a balanced bipartite digraph to be Hamiltonian, arXIV;1506.07949v1 [math. CO] 26 Jun 2015.

[27] D.R. Woodall, Sufficient conditions for circuits in graphs, Proc. Lond. Math. Soc., 24 (1972) 739-755.

[28] K.-M. Zhang, Vertex even-pancylicity in bipartite tournaments, J. Nanjing Univ. Math. Biquarterly 1 (1981) 85-88. 